\title{
Dynamic Analysis of Current Regulators for AC Motors Using Complex Vectors
}

\author{
Fernando Briz del Blanco, Member, IEEE, Michael W. Degner, Associate Member, IEEE, \\ and Robert D. Lorenz, Fellow, IEEE
}

\begin{abstract}
The analysis and design of current regulators for polyphase ac loads is presented using complex vector notation. The ac motor current regulation problem is analyzed by studying both the command tracking and disturbance rejection capability of the current regulator. The use of complex vector notation and the generalization of classical control tools like root locus, frequency-response functions, and dynamic stiffness functions to complex vectors provide a way of comparing the performance of different controller topologies. Limitations in the performance of the synchronous frame proportional and integral current regulator are outlined, and several ways of improving its performance are suggested and investigated.
\end{abstract}

Index Terms - AC current regulators, complex vector analysis, complex vector controls design.

\section{INTRODUCTION}

$\mathbf{T}$ HE synchronous frame proportional and integral (PI) current regulator has become the standard for current regulation of polyphase ac machines due to its capability of regulating ac signals over a wide frequency range [1], [2]. In a reference frame synchronous with the fundamental excitation, the fundamental excitation becomes a dc quantity that is easily regulated to the desired value using a PI controller. Even though the performance characteristics of the synchronous reference frame PI current regulator may seem intuitive, the multiple-input/multiple-output nature of the system makes its performance evaluation difficult.

The representation of ac machines and the analysis of their current regulators can be approached using both scalar and complex vector notation [3]. Although both notations can be used to achieve the same final result, the model of the machine using each notation provides different insight into the control

Paper IPCSD 99-04, presented at the 1998 Industry Applications Society Annual Meeting, St. Louis, MO, October 12-16, and approved for publication in the IEEE TRANSACTIONS ON INDUSTRY APPLICATIONs by the Industrial Power Converter Committee of the IEEE Industry Applications Society. This work was supported by the University of Oviedo, Ford Motor Company, and the Wisconsin Electric Machines and Power Electronics Consortium (WEMPEC) of the University of Wisconsin. Manuscript released for publication January 13, 1999.

F. Briz del Blanco is with the Department of Electrical, Computer and Systems Engineering, University of Oviedo, Gijon 33208, Spain (e-mail: fernando@hecate.etsiig.uniovi.es).

M. W. Degner is with the Department of Mechanical Engineering, University of Wisconsin, Madison, WI 53706 USA (e-mail: degner@cae.wisc.edu).

R. D. Lorenz is with the Department of Electrical and Computer Engineering, University of Wisconsin, Madison, WI 53706 USA (e-mail: lorenz@engr.wisc.edu).

Publisher Item Identifier S 0093-9994(99)07483-6. problem and some solutions can be more intuitively seen using one notation or the other.

The standard matrix or scalar notation does not easily lend itself to classical control tools, like root locus or frequencyresponse functions, other than allowing the use of matrix algebra. The use of complex vector notation simplifies the model of an ac machine from a multiple-input/multiple-output system to an equivalent single-input/single-output complex vector system. The performance of this complex vector model can then be evaluated using generalized forms of classical control tools, like the root locus, frequency-response function (FRF), and dynamic stiffness function (DSF), namely, the complex vector root locus, FRF and DSF.

Complex vectors are systematically used to study the performance of synchronous reference frame current regulators in this paper. Induction motor modeling is presented first. A complex vector-based analysis of the command tracking and disturbance rejection capabilities for the different synchronous frame current regulators is shown to provide increased insight into the induction motor current regulation problem. From this analysis, several improvements are proposed.

\section{Induction Motor Modeling, CuRrent Regulation, AND BACK-EMF DECOUPLING}

The nonlinear state equations governing the electrical and electromagnetic behavior of an induction motor using complex vector notation, with the stator current and the rotor flux as the state variables, are [3]

$$
\begin{aligned}
p i_{q d s}^{s} & =\frac{1}{L_{\sigma s}}\left(v_{q d s}^{s}-R_{s}^{\prime} i_{q d s}^{s}+\frac{L_{m}}{L_{r}} \omega_{b r} \lambda_{q d r}^{s}\right) \\
p \lambda_{q d r}^{s} & =\frac{L_{m}}{L_{r}} R_{r} i_{q d s}^{s}-\omega_{b r} \lambda_{q d r}^{s}
\end{aligned}
$$

where

$$
L_{\sigma s}=L_{s}-\frac{L_{m}^{2}}{L_{r}} R_{s}^{\prime}=R_{s}+\left(\frac{L_{m}}{L_{r}}\right)^{2} R_{r} \omega_{b r}=\frac{R_{r}}{L_{r}}-j \omega_{r} .
$$

The superscript " $s$ " denotes a stationary reference frame and $p$ is the derivative operator.

From a control perspective, it is useful to transform these equations into an excitation frequency synchronous reference frame. The transformation of a generic complex vector quantity $f$ between the stationary and the synchronous reference frame, denoted by the superscript "e," is defined by (3) for the case of a single complex vector quantity and (4) for its 


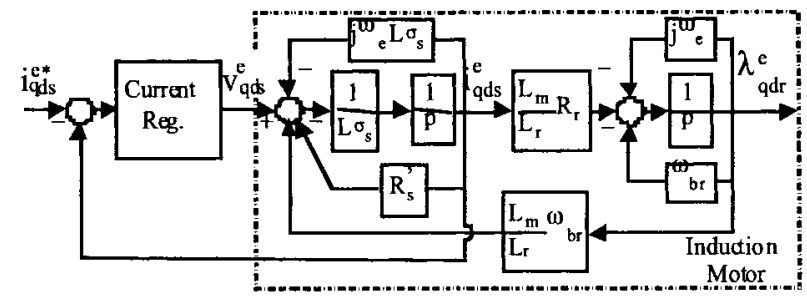

Fig. 1. Complex vector block diagram of a current-regulated induction motor, shown in a synchronous reference frame.

derivative

$$
\begin{aligned}
f_{q d}^{s} & =f_{q d}^{e} e^{j \omega_{e} t} \\
p f_{q d}^{s} & =p\left(f_{q d}^{e} e^{j \omega_{e} t}\right)=\left(p+j \omega_{e}\right) f_{q d}^{e} e^{j \omega_{e} t} .
\end{aligned}
$$

Applying (3) and (4) to (1) and (2), the complex vector equations of the induction motor in a synchronous reference frame (5) and (6) are obtained

$$
\begin{aligned}
p i_{q d s}^{e}= & \frac{1}{L_{\sigma s}}\left(v_{q d s}^{e}-R_{s}^{\prime} i_{q d s}^{e}-j \omega_{e} L_{\sigma s} i_{q d s}^{e}\right. \\
& \left.+\frac{L_{m}}{L_{r}} \omega_{b r} \lambda_{q d r}^{e}\right) \\
p \lambda_{q d r}^{e}= & \frac{L_{m}}{L_{r}} R_{r} i_{q d s}^{e}-\left(\omega_{b r}+j \omega_{e}\right) \lambda_{q d r}^{e} .
\end{aligned}
$$

When using a voltage-source inverter, controlling the stator current (see Fig. 1) simplifies the overall drive control scheme from both a torque control and inverter device protection perspective.

Synchronous frame current regulators have become the industry standard for inverter current regulation. They are preferred because all the electrical variables have dc steadystate values when viewed in a synchronous reference frame. This enables a simple PI regulator to provide zero steadystate error, independent of the synchronous frequency. In spite of this attractive property, the dynamic response of this type of current regulator is far from being ideal, showing a deterioration as the synchronous frequency increases. The dependency of the induction motor, (5), on the synchronous frequency is seen to come from a synchronous frame crosscoupling term

$$
-j \omega_{e} L_{\sigma s} i_{q d s}^{e} \quad \text { or alternately viewed as }-j \omega_{e} i_{q d s}^{e}
$$

and from the electromechanical cross coupling via the speed $(\approx$ synchronous frequency) dependent back-EMF voltage

$$
\frac{L_{m}}{L_{r}} \omega_{b r} \lambda_{q d r}^{e} \text {. }
$$

If these two terms are decoupled from (5), the dependence on the synchronous frequency (and rotor speed) disappears, and the stator voltage equation becomes that of an $R L$ load, enabling simple, fast, and accurate current regulation.

When scalar instead of complex vector notation is used, the transformation of a generic complex vector quantity $f$ from complex vector to scalar notation can be done by taking real and imaginary parts after substituting as shown in [3]

$$
f_{q d}=f_{q}-j f_{d} .
$$

Applying (9) to (5), and assuming (as commonly done for rotor flux field orientation) that the synchronous frame has been aligned with the rotor flux

$$
\lambda_{q d r}^{e}=-j \lambda_{r}^{e}
$$

the equivalent scalar nonlinear state equations (11) and (12) corresponding to the complex vector equation (5) are obtained

$$
\begin{aligned}
& v_{q s}^{e}=L_{\sigma s} p i_{q s}^{e}+R_{s}^{\prime} i_{q s}^{e}+L_{\sigma s} \omega_{e} i_{d s}^{e}+\frac{L_{m}}{L_{r}} \omega_{r} \lambda_{r}^{e} \\
& v_{d s}^{e}=L_{\sigma s} p i_{d s}^{e}+R_{s}^{\prime} i_{d s}^{e}-L_{\sigma s} \omega_{e} i_{q s}^{e}-\frac{L_{m} R_{r}}{L_{r}^{2}} \lambda_{r}^{e} .
\end{aligned}
$$

Different representations of the induction motor are often used [3], [5], depending on how the quantities are rearranged. If, for example, the flux state equation (6) is solved with the current state equation (5), the rotor speed variable can be eliminated with the result, as shown in

$$
\begin{aligned}
& v_{q s}^{e}=L_{\sigma s} p i_{q s}^{e}+R_{s} i_{q s}^{e}+L_{s} \omega_{e} i_{d s}^{e}-\omega_{e} \frac{L_{m}}{R_{r}} p \lambda_{r}^{e} \\
& v_{d s}^{e}=L_{\sigma s} p i_{d s}^{e}+R_{s} i_{d s}^{e}-L_{\sigma s} \omega_{e} i_{q s}^{e}+\frac{L_{m}}{L_{r}} p \lambda_{r}^{e} .
\end{aligned}
$$

This representation is no longer a set of state equations, since two state derivatives appear in each equation. Nevertheless, the equations do allow some insight if some assumptions are made about the rate of change of the states. If the rotor flux dynamics are assumed to be much slower than the stator current dynamics, the rotor flux derivative terms are small and, in certain cases, can be neglected [3], [5], [7].

It should be noted that the two rotor flux derivative terms have very distinct coefficients. One is nearly constant, and one is linearly dependent on excitation frequency. Thus, the assumption is most valid for low excitation frequencies.

If the rotor flux dynamic terms can be safely neglected, then the resulting stator voltage equations may be viewed as approximate state equations for the stator current. The synchronous frequency cross coupling now appears as in (15) and (16), which is of a very different form than (7) and (8)

$$
\begin{aligned}
& L_{\sigma s} \omega_{e} e_{q s}^{e} \\
& L_{s} \omega_{e} i_{d s}^{e} .
\end{aligned}
$$

If these two terms are decoupled from (13) and (14), respectively, and the rotor flux derivative terms can be safely neglected, then approximately decoupled control of the $q$ - and $d$-axes currents can be obtained. The resulting approximate stator voltage equation becomes that of an $R L$ load, which is an approximation to the exact decoupling of (7) and (8). Note that the resistance of the remaining $R L$ load in (13) and (14) does not correspond to the resistance in (5) once decoupling has been carried out.

Exact decoupling of (7) and (8) can be viewed as addressing two separate cross-coupling issues. It is instructive to first understand that the cross coupling in (7) results solely from the synchronous frame transform. This can be demonstrated via (17) and (18), which represent a three-phase symmetric 
$R L$ load in stationary and the synchronous reference frames, respectively

$$
\begin{aligned}
& p i_{q d s}^{s}=\frac{1}{L}\left(v_{q d s}^{s}-R i_{q d s}^{s}\right) \\
& p i_{q d s}^{e}=\frac{1}{L}\left(v_{q d s}^{e}-R i_{q d s}^{e}-j \omega_{e} L i_{q d s}^{e}\right) .
\end{aligned}
$$

It is seen that, by transforming an $R L$ load to the synchronous reference frame, an identical cross-coupling term to that present in the induction motor (7) is created. This term, therefore, is a characteristic of $R L$ loads when transformed, and not of the induction motor, in particular. Because the cross coupling can be expressed as $-j \omega_{e}$, appropriately formed decoupling of (7) requires no parameters and can be performed exactly.

In contrast to this, the cross coupling represented by (8) is the effect of back EMF, i.e., rotor flux and rotor velocity, on the stator current. This electromechanical cross coupling could be viewed as a disturbance if the induction motor were modeled as an $R L$ load. From a control systems perspective, however, if approximate decoupling of this electromechanical cross coupling can be achieved, the overall system dynamics are improved, and current regulator properties will be nearly speed invariant.

Because the approximate decoupling solution is less insightful in its terms and is also limited to low excitation frequencies (and/or constant rotor flux), it is considered less attractive than the exact decoupling solution as a global current regulation strategy. Therefore, using (5), or the equivalent scalar notation (11) and (12), to approach the induction motor current regulation problem is considered more appropriate and is used for the remainder of this paper.

The different nature and source of the cross coupling in (7) and (8) suggest that they be considered separately in the controller design. This is done in the following sections.

\section{EFFECTS OF Synchronous Frame Cross COUPLING ON CURRENT REGULATION}

If the rotor-flux-dependent term (back EMF) (8) is perfectly decoupled from the stator voltage equation of the induction motor (5), the equation reduces to that of a simple $R L$ load (18). Using this assumption, it is possible to look at the effects of the synchronous frame cross-coupling term (7) on the performance of current regulators for induction motors with the simplified model of an $R L$ load [4].

The complex vector model of an $R L$ load in the synchronous reference frame (18) is seen to have a single, asymmetric, complex pole located at $-R / L-j \omega_{e}$. The complex vector block diagram of the $R L$ load with a synchronous frame PI current regulator is shown in Fig. 2.

The performance of the classical synchronous frame PI current regulator was analyzed by applying it to a three-phase $R L$ load with the parameters shown in Table I.

The current regulator was tuned by selecting a controller zero approximately equal to the break frequency of the $R L$ load, i.e., $K_{i} / K_{p}=R / L$. The controller gain was selected to achieve a relatively low bandwidth of $200 \mathrm{~Hz}$, so that systematic transient errors would be more easily observed.

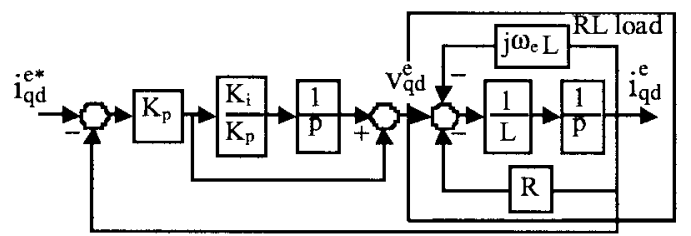

Fig. 2. Complex vector block diagram of an $R L$ load with a synchronous frame PI current regulator, shown in the synchronous reference frame.

TABLE I

$R L$ LOAD PARAMETers

\begin{tabular}{lr}
\hline$R$ & $1.1 \Omega$ \\
$L$ & $3.7 \mathrm{mH}$ \\
\hline
\end{tabular}
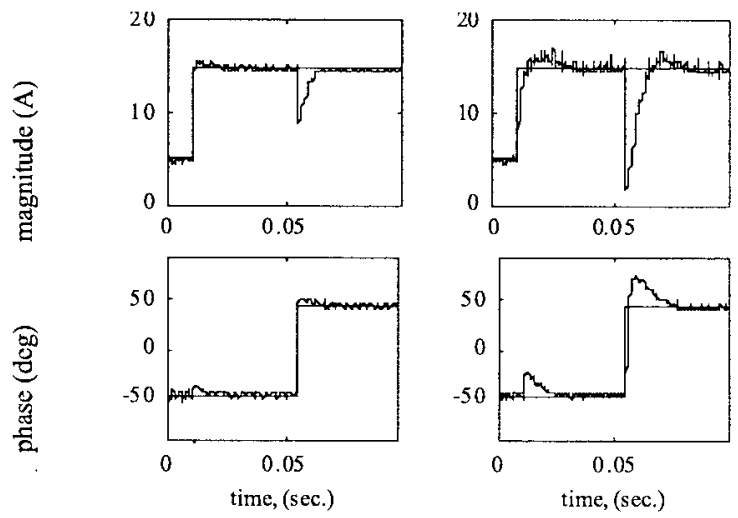

Fig. 3. Commanded and experimental current magnitude and phase step responses for an $R L$ load with a synchronous frame PI current regulator (200-Hz bandwidth), shown in the synchronous reference frame.

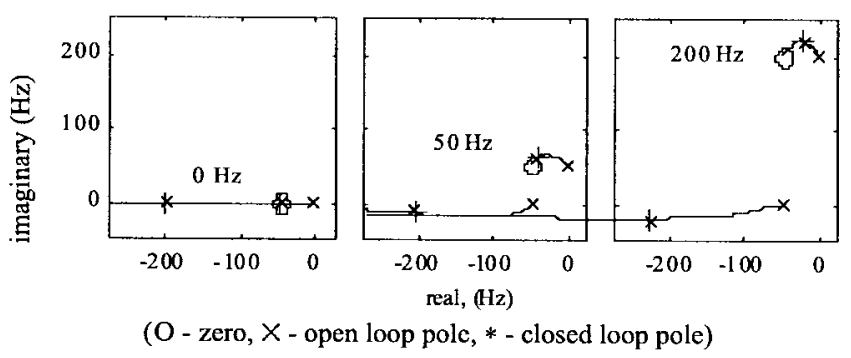

Fig. 4. Complex vector root locus of an $R L$ load with a synchronous frame PI current regulator (200-Hz bandwidth), shown in the stationary reference frame $\left(f_{e}=0,50\right.$, and $\left.200 \mathrm{~Hz}\right)$.

An overlay of the commanded and experimental system response for a magnitude and a phase step, with constant input synchronous frequencies of 50 and $200 \mathrm{~Hz}$, is shown in Fig. 3. Serious degradation in the transient performance is apparent as the synchronous frequency increases.

The complex vector root locus can also be plotted, as shown in Fig. 4 for three different synchronous frequencies. The complex vector root locus, as the scalar root locus, follows the magnitude and angle conditions. Nevertheless, because the inputs and outputs are no longer real numbers but complex vectors, it is possible to get complex, asymmetric poles and zeros, i.e., the root locus does not have to be symmetric with 


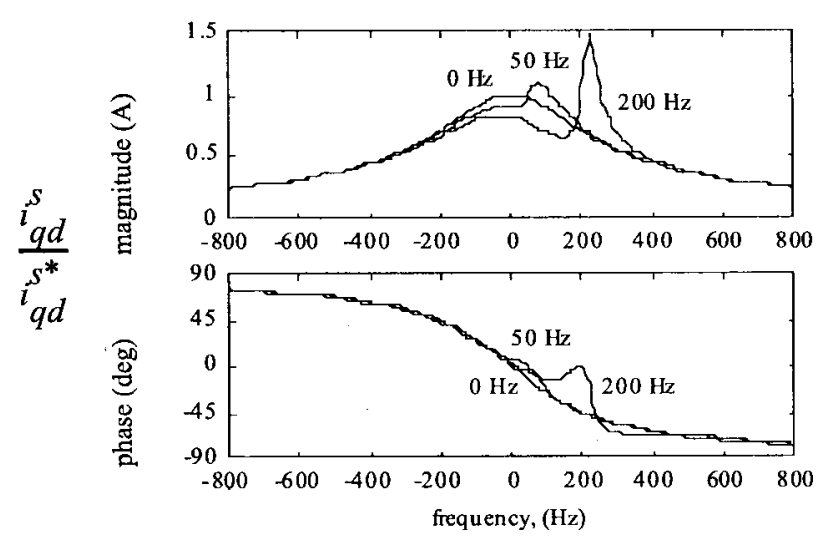

Fig. 5. Complex vector FRF of an $R L$ load with a synchronous frame PI current regulator (200-Hz bandwidth), shown in the stationary reference frame $\left(f_{e}=0,50\right.$, and $\left.200 \mathrm{~Hz}\right)$.

respect to the real axis. The root locus was obtained using the standard root locus functions in the Matlab controls systems toolbox.

From Fig. 4, it is seen that, at low frequencies, the controller zero approximately cancels the plant pole. This allows the response of the system to be dominated by the faster closedloop pole, placed at the desired $200-\mathrm{Hz}$ bandwidth. For higher synchronous frequencies, the controller zero interacts more with the pole added by the controller. The resulting slower root moves progressively closer to the imaginary axis away from the zero, with increasing overshoot expected. The complex vector transfer function describing the system is given by

$$
\frac{i_{q d}^{s}}{i_{q d}^{s *}}=\frac{K_{p} s+K_{i}-j K_{p} \omega_{e}}{L s^{2}+\left(K_{p}+R-j \omega_{e} L\right) s+K_{i}-j \omega_{e}\left(K_{p}+R\right)}
$$

The complex vector FRF, shown in Fig. 5, can be calculated from this transfer function. The asymmetric root locus about the real axis gives rise to an FRF asymmetric for positive and negative frequencies. It is noted that all of the FRF's shown in Fig. 5 have a unity gain and zero phase shift at the synchronous frequency. However, at frequencies away from the synchronous frequency, there is significant distortion in the FRF.

It is important to understand the meaning of the FRF at frequencies other than the synchronous frequency. The synchronous frequency is the steady-state fundamental component. Both disturbances and changes in the command trajectory simultaneously excite the system with a wide range of frequency content. The FRF shows how the system responds to the frequency content that is not at the synchronous frequency.

From this analysis, the time response in Fig. 3 can be explained. Even though the commanded synchronous frequency remained constant, magnitude and phase steps in the commanded current introduced transient content at frequencies centered on the synchronous frequency. Thus, the transient response of the current regulator depends on its capability to regulate beyond the synchronous frequency.

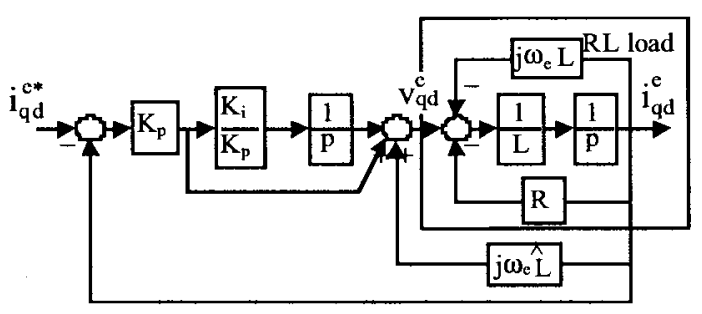

Fig. 6. Complex vector block diagram of an $R L$ load with a cross-coupling decoupling (via state feedback) synchronous frame PI current regulator, shown in the synchronous reference frame.

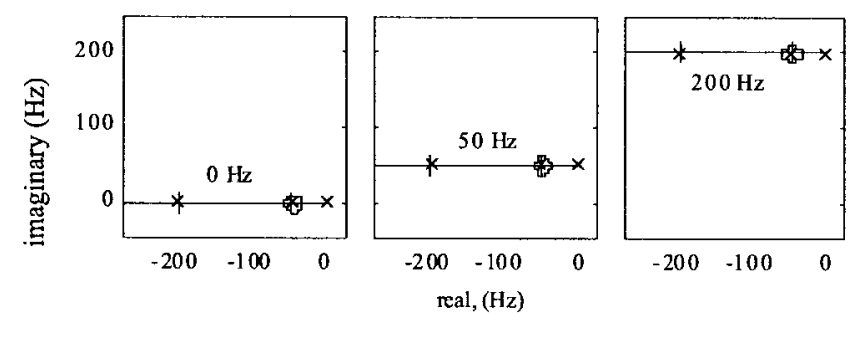

(O - zero, $x$ - open loop pole, * - closed loop pole)

Fig. 7. Complex vector root locus of an $R L$ load with a cross-coupling decoupling (via state feedback) synchronous frame PI current regulator (200-Hz bandwidth), shown in the stationary reference frame $\left(f_{e}=0,50\right.$, and $200 \mathrm{~Hz}$ ).

\section{IMPROVED CURRENT REGUlATOR DESIGN BY DECOUPLING OF SYNCHRONOUS Frame CROSS COUPLING}

An ideal synchronous reference frame current regulator would have a time response independent of the synchronous frequency when viewed in the synchronous reference frame. Such a regulator would have a complex vector FRF with a shape that does not vary with the synchronous frequency. Instead, the center of the FRF shape would just shift so that it is always symmetric about the synchronous frequency. To achieve this will require decoupling the effect of the synchronous frequency cross coupling. There are two possibilities for decoupling: 1) state feedback decoupling and 2) symmetric cross coupling.

\section{A. Synchronous Frame PI Current Regulator with Cross-Coupling Decoupling via State Feedback}

One way of modifying the synchronous frame PI current regulator to achieve the desired response is to decouple the cross coupling caused by the term $j \omega_{e} L$ in (18). The block diagram of the cross-coupling decoupling form of the synchronous frame PI current regulator is in Fig. 6 [4], [5]. The effect of the cross-coupling decoupling is to move the pole of the plant from $-R / L-j \omega_{e}$ to $-R / L$ in the synchronous reference frame, which makes it possible to directly cancel it using the real zero added by the controller. The resulting complex vector root locus for the cross-coupling decoupling synchronous frame PI current regulator is shown in Fig. 7 for three different synchronous frequencies.

It should be noted that, by removing the plant cross coupling in the synchronous frame, the system in the stationary frame will now be cross coupled. This is because cross coupling 


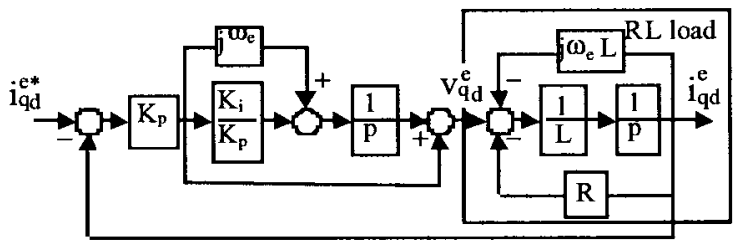

Fig. 8. Complex vector block diagram of an $R L$ load with a complex vector synchronous frame PI current regulator, shown in the synchronous reference frame.

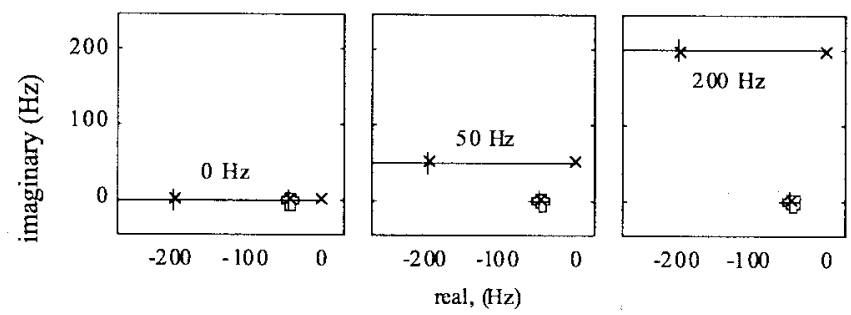

(O - zero, $\times$ - open loop pole, $*$ - closed loop pole)

Fig. 9. Complex vector root locus for an $R L$ load with a complex vector synchronous frame PI current regulator (200-Hz bandwidth), shown in the stationary reference frame $\left(f_{e}=0,50\right.$, and $\left.200 \mathrm{~Hz}\right)$.

occurs with the inverse transform of an uncoupled synchronous frame system to the stationary frame. Thus, in the stationary frame root locus, the controller zero appears to move with the plant pole and both are tied to the synchronous frequency.

\section{B. Complex Vector Synchronous Frame PI Current Regulator with Symmetric Cross Coupling}

Instead of moving the pole of the plant to the location of the controller zero, the controller zero can be moved to the location of the plant pole by modifying the controller structure as shown in Fig. 8 to symmetrically cross couple the controller with the synchronous frequency term [4], [6]. This form of the synchronous frame PI current regulator is called the complex vector synchronous frame PI current regulator [4].

This design is directly analogous to the classical control pole/zero cancellation methodology, with the only difference being the use of complex vectors, which allows the placement of the controller zero off the real axis. The resulting complex vector root locus is shown in Fig. 9 for three different synchronous frequencies.

It should be noted that, by symmetrically cross coupling the controller (and the plant) in the synchronous frame, both the controller and the plant will be decoupled in the stationary frame. Thus, in the stationary frame root locus, the controller zero appears fixed with the plant pole and neither are tied to the synchronous frequency.

It should further be noted that the symmetric cross coupling has no physical parameters, which is consistent with the fact that the origin of this cross coupling is solely from the synchronous frame transform.

If the parameter estimates are correct, and both modified current regulators have the same controller PI gains, the

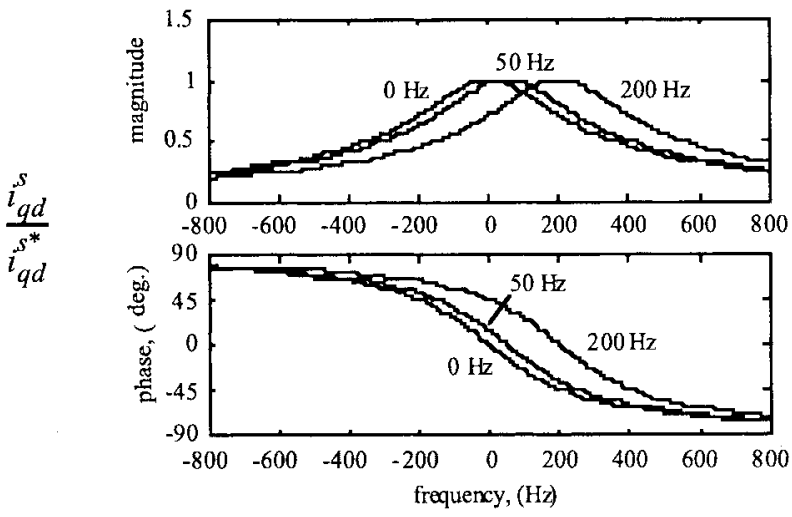

Fig. 10. Complex vector FRF of an $R L$ load for either a cross-coupling decoupling or complex vector synchronous frame PI current regulator $(200-\mathrm{Hz}$ bandwidth), shown in the stationary frame $\left(f_{e}=0,50\right.$, and $\left.200 \mathrm{~Hz}\right)$.
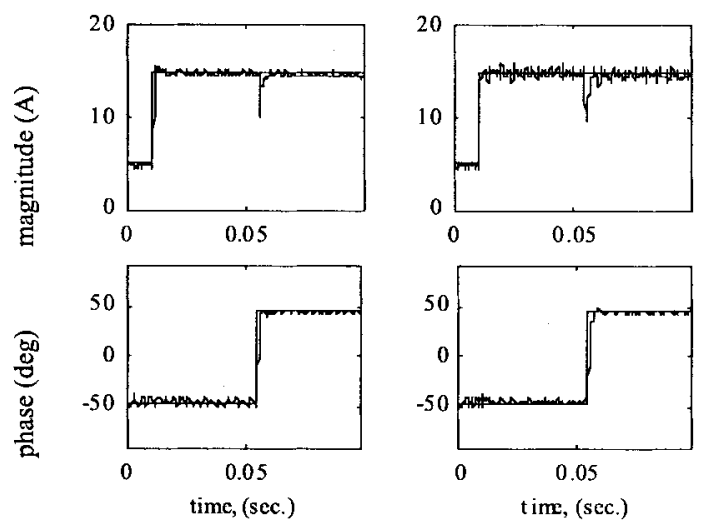

Fig. 11. Commanded and experimental current magnitude and phase step response for an $R L$ load with a complex vector synchronous frame PI current regulator $(200-\mathrm{Hz}$ bandwidth), shown in the synchronous frame.

complex vector FRF's for the two current regulators are identical and shown in Fig. 10 [4]. The shape of the complex vector FRF is independent of the synchronous frequency and symmetric with respect to it. Fig. 11 shows the step response for the complex vector synchronous frame PI current regulator. (The cross-coupling decoupling controller has nearly identical characteristics and, thus, is not shown.) The time response is seen now to correspond to the tuned bandwidth independent of the synchronous frequency.

The decreased parameter sensitivity of the complex vector form of the synchronous frame PI current regulator in comparison with the cross-coupling decoupling via state feedback was demonstrated in [4]. The main reason for this difference is the beneficial impact of symmetric cross coupling in achieving pole-zero cancellation independent of the synchronous frequency.

\section{DYNAMIC STIFFNESS ANALYSIS FOR SYNCHRONOUS FRAME PI CURRENT REGULATORS}

The simplification of the induction motor to an $R L$ load could also be done by considering the back-EMF term (8), as a disturbance to the stator voltage equation (5), as shown in Fig. 12, instead of assuming perfect decoupling. 


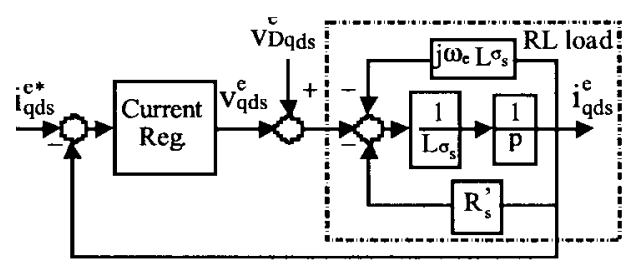

Fig. 12. Representation of the induction motor by an $R L$ load with disturbance input shown in a synchronous reference frame.

(a)

(b)

(c)

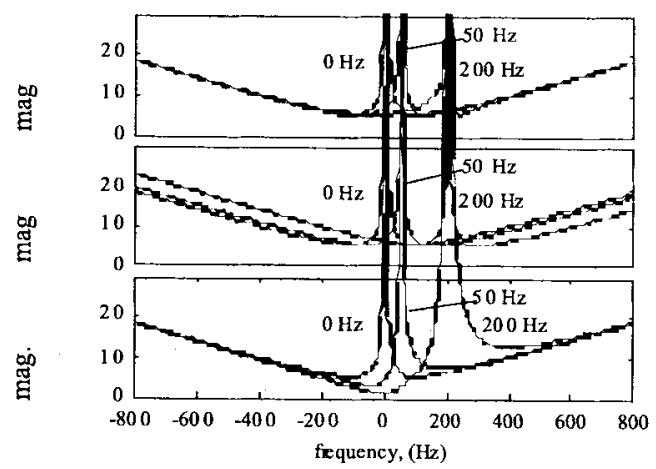

Fig. 13. Dynamic stiffness analysis for an $R L$ load with (a) classical, (b) cross-coupling decoupling, and (c) complex vector synchronous PI current regulators $\left(200-\mathrm{Hz}\right.$ bandwidth), shown in the stationary frame $\left(f_{e}=0,50\right.$, and $200 \mathrm{~Hz}$ ).

In this case, the back EMF would be a disturbance to the system that would need to be compensated for by the current regulator. This suggests that a study of the disturbance rejection capability of the different current regulator designs by mean of the DSF generalized to complex vectors will provide increased insights in analyzing the effect of back EMF.

Substituting the "current regulator" block in Fig. 12 with the different current regulators transfer functions, the following complex vector DSF's, i.e., disturbance versus output transfer functions, are obtained for the classical (20), crosscoupling decoupling (21), and complex vector (22) current regulators. Separating the physical parameters instead of the usual pole-zero representation was considered to provide more insight

$$
\begin{aligned}
& \frac{V_{D q d}^{s}}{i_{q d}^{s}}=L_{\sigma s} s+R_{s}^{\prime}+K_{p}+\frac{K_{i}}{s-j \omega_{e}} \\
& \frac{V_{D q d}^{s}}{i_{q d}^{s}}=L_{\sigma s} s+R_{s}^{\prime}+K_{p}-j \omega_{e} \hat{L}_{\sigma s}+\frac{K_{i}}{s-j \omega_{e}} \\
& \frac{V_{D q d}^{s}}{i_{q d}^{s}}=L_{\sigma s} s+R_{s}^{\prime}+\frac{K_{p} s}{s-j \omega_{e}}+\frac{K_{i}}{s-j \omega_{e}}
\end{aligned}
$$

It is noted that the dynamic stiffness has units of impedance where, in this case, high impedance with respect to the backEMF "disturbance" voltage would be preferred.

These functions are represented in Fig. 13 (only magnitudes are shown). As was the case for the complex vector FRF, the disturbance input is not a sinusoidal signal, but a rotating vector, and because positive and negative frequencies are needed, a linear scale has to be used for the frequency axis.
For $s=j \omega_{e}$, i.e., the disturbance voltage vector rotating at the synchronous frequency, all three current regulator designs provide infinite dynamic stiffness, therefore, the current regulation is not affected by the disturbance input, which agrees with the zero steady-state error property. At frequencies other than the synchronous frequency, the DSF shows how much the current regulation will be affected. The minimum in each curve shows the frequency for which the current regulator will be weakest (lowest impedance), and how weak it will be. By comparing the three DSF's one can conclude the following.

- For $\omega_{e}=0$ (dc excitation), all three regulators behave the same.

- With correct parameter estimates, the cross-coupling decoupling DSF remains invariant with the synchronous frequency.

- The classical design DSF moves its minimum magnitude frequency closer to the synchronous frequency as the synchronous frequency increases, which could result in low-frequency oscillations when viewed in a synchronous reference frame.

- As the synchronous frequency increases, the complex vector design shows a reduced dynamic stiffness at low frequencies, but presents an increased dynamic stiffness at frequencies near the synchronous frequency.

For this DSF analysis to become meaningful for the induction motor, it is necessary to determine which region of the spectrum will be excited by disturbances such as back EMF.

\section{EFFECT OF THE BACK EMF ON THE StATOR CURRENT}

Rewriting (8) as shown in (23), the disturbance due to the back EMF on the stator current is seen to depend on the rotor flux and the rotor speed

$$
V_{D q d}^{e}=\frac{L_{m}}{L_{r}} \omega_{b r} \lambda_{q d r}^{e}=\frac{L_{m}}{L_{r}}\left(\frac{R_{r}}{L_{r}}-j \omega_{r}\right) \lambda_{q d r}^{e} .
$$

For rotor-flux-oriented drives, where efficiency and thermal limits are not important factors, it is not uncommon to keep the rotor flux constant below rated speed. This causes $V_{D q d}^{e}$ to vary proportional to the rotor speed, which means that it is very dependent on the inertia and acceleration properties of the motor and load. For modern servo drives, these dynamics are often as fast as the electrical dynamics and play a significant role in the system dynamics.

The rotor flux can also vary dynamically due to estimation errors, since rotor flux is often regulated by feedback from flux observers. Although the dynamic analysis of the different flux observer designs is beyond the scope of this paper [10], [11], a brief study for the case of a current-model-based flux observer is presented.

The transfer function for the current-model-based flux observer as shown in (24) is obtained from (6), with $\tau_{r}$ denoting the rotor time constant and $\omega_{s}=\omega_{e}-\omega_{r}$ the slip

$$
\frac{\lambda_{q d r}^{e}}{i_{q d s}^{e}}=\frac{L_{m}}{\tau_{r}\left(s+j \omega_{s}\right)+1} .
$$




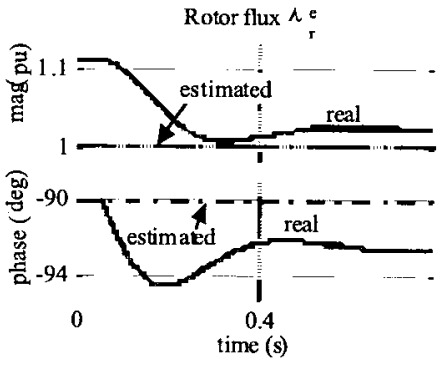

(a)

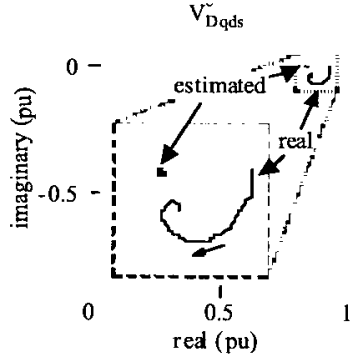

(b)
Fig. 14. Current-model-based flux observer transient response with a $q$-axis-current rated step command, with a $10 \%$ underestimation in the flux observer inductances $\left(\omega_{r}=1500 \mathrm{r} / \mathrm{min}\right.$, infinite inertia). (a) Actual and estimated flux. (b) Actual and estimated back EMF. Shown in a reference frame synchronous with the estimated flux.

Fig. 14 shows a simulation of a rated $q$-axis-current step with a rotor speed equal to three times the rated speed, infinite inertia, and a $10 \%$ underestimation in the flux observer inductance. The $d$ axis is aligned with the estimated rotor flux at $-90^{\circ}$ according to (10). Fast current regulators will keep the estimated rotor flux magnitude constant and equal to the commanded value, but variations both in the magnitude and the phase of the actual flux are observed. The steady-state error of such flux observers is known to depend on the slip [10], while its dynamics when the slip changes are deduced from (24) to oscillate at the slip frequency and damped with the rotor time constant, as seen in Fig. 14(b) [11].

It can be concluded that, when errors in the parameters exist, the estimated and actual flux will also have errors between them. Because of this, the dynamic stiffness near the synchronous frequency will determine the expected performance for the different current regulator designs with respect to the flux-induced back-EMF dynamics. The dynamic stiffness in the baseband region, on the other hand, is dominated by the mechanical dynamics of the motor and load. Since the load is unknown, this region should be made as stiff as possible to best reject real disturbances. This results in the following conclusions about the DSF of the three current regulator topologies.

- The classical synchronous frame PI current regulator shows reduced dynamic stiffness near the synchronous frequency as the synchronous frequency increases, which makes it more sensitive to rotor flux disturbances. It also has modest dynamic stiffness in the baseband region as needed to minimize the effects of the mechanical dynamics.

- The cross-coupling decoupling design keeps invariant its dynamic stiffness function. The influence of the rotor flux on the stator current regulation is independent of the synchronous frequency. This design has the best baseband dynamic stiffness of the three designs.

- The complex vector design shows an increased dynamic stiffness at frequencies near the synchronous frequency as the synchronous frequency increases, which suggests that it should not be affected by the rotor flux. On the other hand, this controller, as configured, has the lowest dynamic stiffness in the baseband region.

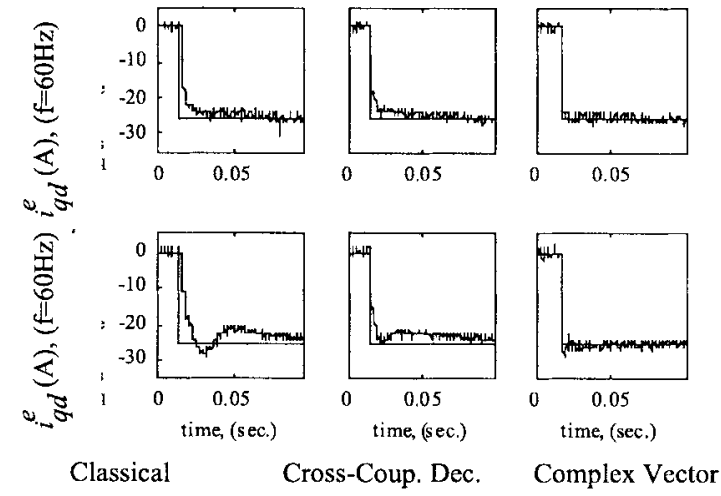

(a)
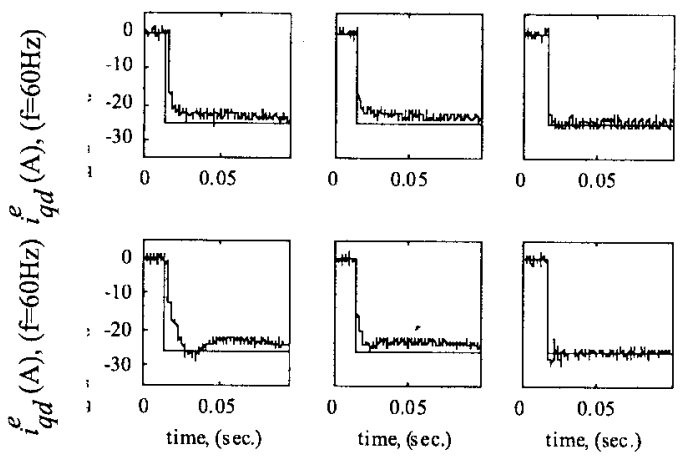

Classical

Cross-Coup. Dec.

Complex Vector

(b)

Fig. 15. Experimental $q$-axis-current step response for and induction motor with three different forms on synchronous PI current regulators: (a) with and (b) without back-EMF decoupling $\left(200-\mathrm{Hz}\right.$ bandwidth, $f_{e}=60$ and $160 \mathrm{~Hz}$ ), shown in the synchronous reference frame.

\section{COMMAND RESPONSE COMPARISONS FOR SYNCHRONOUS FRAME PI CURRENT REGULATORS}

Fig. 15 shows the $q$-axis-current step response of an induction motor for the three different current regulator designs with and without back-EMF decoupling implemented. The induction motor had the following parameter values: $R_{s}^{\prime}=$ $0.82 \Omega, L_{\sigma s}=5.5 \mathrm{mH}, L_{m}=148 \mathrm{mH}, L_{r}=150 \mathrm{mH}$, $R_{r}=0.36 \Omega$, power rating $=6 \mathrm{~kW}$, and four poles. The observed transient response, including settling time for each current regulator design, validates the response properties predicted from the previous analysis.

\section{IMPROVING THE BASEBAND DYNAMIC STIFFNESS OF COMPLEX VECTOR SYNCHRONOUS FRAME PI REGULATORS}

Despite the relatively good command tracking performance of the complex vector synchronous PI current regulator in comparison to the classical design, its low stiffness near dc (baseband) would not be tolerable. Increasing the bandwidth, i.e., $K_{p}$, for any of the current regulator designs increases their dynamic stiffness. It should be noted that, for the classical and cross-coupling decoupling designs, the dynamic stiffness (impedance) provided by the gain $K_{p}$ is independent of the frequency, acting like a resistance. 


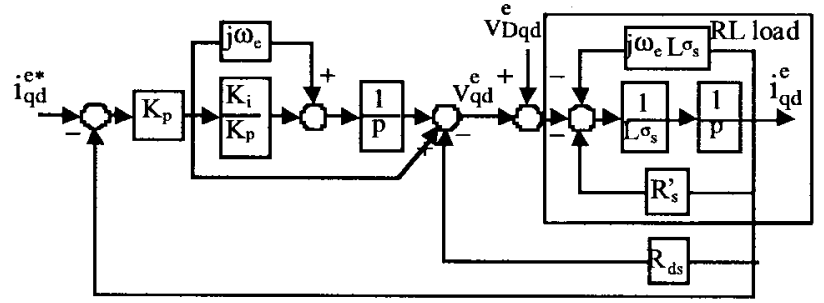

Fig. 16. Representation of the induction motor by an $R L$ load with a complex vector synchronous frame PI current regulator with "active" resistance, shown in the synchronous reference frame.

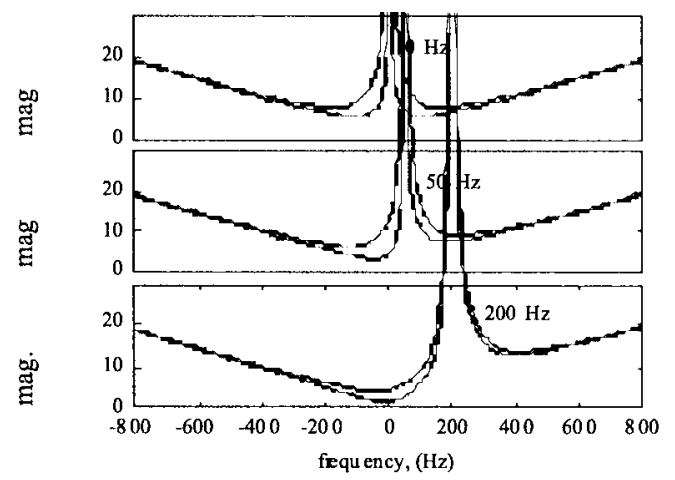

Fig. 17. DC stiffness increase for the complex vector synchronous frame PI current regulator by adding "active" resistance. $R_{d s}=0$ (dashed), $R_{d s}=3 R$ (solid) (200-Hz bandwidth), shown in the stationary reference frame $\left(f_{e}=\right.$, 0 , 50, and $200 \mathrm{~Hz}$ ).

For the case of the complex vector design, it is seen from (22) that the gain $K_{p}$ provides maximum dynamic stiffness near the synchronous frequency, but reduced dynamic stiffness near dc. Its dynamic stiffness at frequencies near dc is provided entirely by the physical resistance in the system.

Modifying the complex vector current regulator design as shown in Fig. 16 can increase its baseband dynamic stiffness. With this design, the DSF in (25) is obtained, which is represented in Fig. 17. It is noted that the gain $R_{d s}$ has the same effect in (25) as the gain $K_{p}$ had in (20) and (21)

$$
\frac{V_{D q d}^{s}}{i_{q d}^{s}}=L_{\sigma s} s+R_{s}^{\prime}+R_{d s}+\frac{K_{p} s}{s-j \omega_{e}}+\frac{K_{i}}{s-j \omega_{e}} .
$$

It can be demonstrated that, if the zero of the regulator is calculated according to (26), the command tracking properties of the modified regulator remain unchanged, i.e., the gain $K_{p}$ is the same, the FRF (and, therefore, the bandwidth) remaining as shown in Fig. 10

$$
K_{i} / K_{p}=\left(R_{s}^{\prime}+R_{d s}\right) / L_{\sigma s} .
$$

Fig. 18 shows the $q$-axis-current step response for the complex vector current regulator design with and without the $R_{d s}$ gain, demonstrating the improved performance with $R_{d s}$.

One conclusion that might be reached is that increasing $R_{d s}$ can increase the de dynamic stiffness without limit. This would be incorrect from at least two perspectives. From one perspective, it can be seen that $R_{d s}$ acts as an increased loop

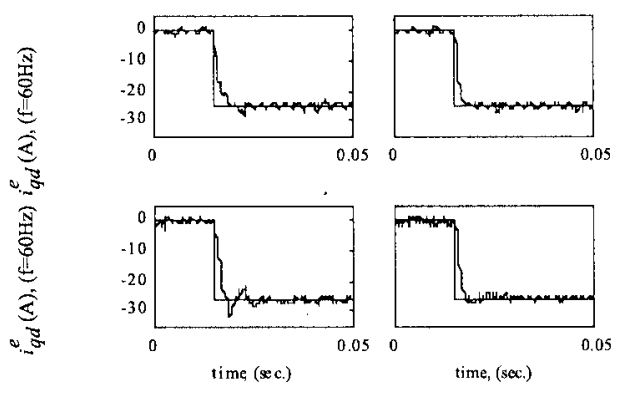

Fig. 18. Experimental $q$-axis-current step response for an induction motor with a complex vector synchronous frame PI current regulator with two different tuning methods, without back-EMF decoupling (200-Hz bandwidth, $f_{e}=60$ and $160 \mathrm{~Hz}$ ), shown in the synchronous reference frame.

gain. Thus, the actual root for the innermost loop is much higher than the command bandwidth. This inner bandwidth is inherently limited by switching frequency and by quantization noise on the current signal. From a second perspective, it can be noted that as this $R_{d s}$ gain increases, the pole-zero cancellation according to (26) moves left on the complex plane, toward higher frequencies, making it more sensitive to noise. Nevertheless, for the $200-\mathrm{Hz}$ command bandwidth, values for $R_{d s}$ as high as ten times the stator transient resistance were implemented experimentally without any noticeable deterioration in the response due to noise.

\section{CONCLUSION}

This paper has attempted to make two contributions: 1) development of complex vector methods for current regulator design and analysis and 2) a comparison of current regulator performance using the developed analysis tools.

From the paper's theoretical and experimental results, several important conclusions can be reached.

- The performance of induction motor synchronous frame current regulators is affected by the synchronous frequency through two distinct ways: 1) the cross coupling intrinsic to the transformation of $R L$ loads to a rotating reference frame and 2) the electromechanical cross coupling due to back EMF.

- The command tracking performance of the classical synchronous frame PI current regulator degrades as the synchronous frequency approaches the current regulator bandwidth. This regulator design has also been shown to have an increased sensitivity to back EMF.

- The cross-coupling decoupling and complex vector synchronous frame PI designs provide, even with incorrect parameters, improved command tracking properties.

- The reduced parameter sensitivity for command tracking and increased dynamic stiffness near the synchronous frequency of the complex vector current regulator make it a viable option for current regulation at high synchronous frequencies. Its degraded dynamic stiffness at low frequencies can be overcome by the addition of appropriate state feedback with active resistance properties.

- The additional computational requirements of the improved synchronous frame current regulators are minimal. 


\section{REFERENCES}

[1] C. D. Schauder and R. Caddy, "Current control of voltage-source inverters for fast four-quadrant drive performance," IEEE Trans. Ind. Applicat., vol. IA-18, pp. 163-171, Mar./Apr. 1982.

[2] T. R. Rowan and R. L. Kerkman, "A new synchronous current regulator and an analysis of current-regulated PWM inverters," IEEE Trans. Ind. Applicat., vol. IA-22, pp. 678-690, July/Aug. 1986.

[3] D. W. Novotny and T. A. Lipo, Vector Control and Dynamics of AC Drives. New York: Oxford Univ. Press, 1996.

[4] F. Briz del Blanco, M. W. Degner, and R. D. Lorenz, "Analysis and design of current regulators using complex vectors," in Conf. Rec. IEEE-IAS Апnи. Meeting, New Orleans, LA, 1997, pp. 1504-1511.

[5] L. J. Garces, "Current control of field oriented induction motor drives," in IEEE Tutorial: Microprocessor Control of Motor Drives and Power Converters. New York: IEEE Press, 1995, pp. 5.1-5.46.

[6] L. Harnefors and H. P. Nee, "Model-based control of AC machines using the internal model control method," IEEE Trans. Ind. Applicat. vol. 34, pp. 133-141, Jan./Feb. 1998.

[7] J. Jung, S. Lim, and K. Nam, "PI type decoupling control scheme for high speed operation of induction motors," in Proc. IEEE PESC'97, 1997, pp. 1082-1085.

[8] D. Y. Ohm and R. J. Oleksuk, "On practical digital current regulator design for PM synchronous motor drives," in Proc. IEEE APEC'98, 1998, pp. 56-63.

[9] R. D. Lorenz and D. B. Lawson, "Performance of feed forward current regulators for field oriented induction machine controllers," IEEE Trans. Ind. Applicat., vol. 23, pp. 597-602, July/Aug. 1987.

[10] P. L. Jansen and R. D. Lorenz, "A physically insightful approach to the design and accuracy assessment of flux observers for field oriented IM drives," IEEE Trans. Ind. Applicat., vol. 30, pp. 101-110, Jan./Feb. 1994.

[11] G. C. Verghese and S. R. Sanders, "Observers for flux estimation in induction machines," IEEE Trans. Ind. Electron., vol. 35, pp. 85-94, Feb. 1988.

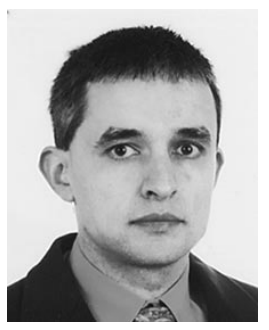

Fernando Briz del Blanco (A'96-M'99) received the M.S. and Ph.D. degrees from the University of Oviedo, Gijon, Spain, in 1990 and 1996, respectively.

From June 1996 to March 1997, he was a Visiting Researcher at the University of Wisconsin, Madison. $\mathrm{He}$ is currently an Assistant Professor in the Electrical Engineering Department, University of Oviedo. His areas of interest include control systems, highperformance ac drives control, sensorless control, and digital signal processing.

Dr. Briz del Blanco was the recipient of a Prize Paper Award from the Industrial Power Converter Committee of the IEEE Industry Applications Society.

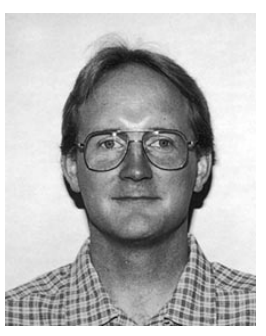

Michael W. Degner (S'95-A'98) received the B.S., M.S., and Ph.D. degrees in mechanical engineering, with a focus in electromechanics and control, from the University of Wisconsin, Madison, in 1991, 1993, and 1998, respectively.

$\mathrm{He}$ is currently with the Ford Research Laboratory, Ford Motor Company, Dearborn, MI, where his research is focused on the use of power electronics in automobile applications. His interests include control systems, machine drives, electric machines, power electronics, and electromechanics.

Dr. Degner received the Second Prize Paper Award from the Industrial Drives Committee and the Third Prize Paper Award from the Industrial Power Converter Committee at the 1997 IEEE Industry Applications Society Annual Meeting.

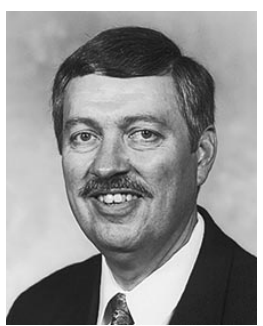

Robert D. Lorenz (S'83-M'84-SM'91-F'98) received the B.S., M.S., and Ph.D. degrees from the University of Wisconsin, Madison, in 1969, 1970, and 1984, respectively.

Since 1984, he has been a member of the faculty in both the Mechanical Engineering and the Electrical and Computer Engineering Departments, University of Wisconsin, where he is the Consolidated Papers Foundation Professor of Controls Engineering. In this position, he acts as Codirector of the Wisconsin Electric Machines and Power Electronics Consortium. He is also an active consultant to many organizations. He was a Visiting Research Professor in the Electrical Drives Group, Catholic University of Leuven, Leuven, Belgium, in the summer of 1989 and in the Power Electronics and Electrical Drives Institute, Technical University of Aachen, Aachen, Germany, in the summers of 1987, 1991, 1995, 1997, and 1999. In 1969-1970, he conducted research for the M.S. thesis at the Technical University of Aachen. From 1972 to 1982, he was a member of the research staff at the Gleason Works, Rochester, NY. His current research interests include sensorless electromagnetic motor/actuator technologies, realtime signal processing and estimation techniques, precision multi-axis motion control, and ac drive and high-precision machine control technologies.

Dr. Lorenz serves currently as the Secretary of the IEEE Industry Applications Society (IAS) and immediate Past Chair of the IAS Awards Department. $\mathrm{He}$ is a member and Past Chairman of the IAS Industrial Drives Committee and a member of the IAS Electric Machines, Industrial Power Converter, and Industrial Automation and Control Committees. He is a Registered Professional Engineer in the States of New York and Wisconsin. He is also a member of the American Society of Mechanical Engineers, Instrument Society of America, and Society of Photo-Optical Instrumentation Engineers. 